\title{
Mechanical behavior of sand under high pressure and high strain rate
}

\author{
S. Abdel-Malek, L.W. Meyer, and N. Herzig \\ Nordmetall Impact Dynamics GmbH, Hauptstrasse 16, 09221 Adorf, Germany, www.nordmetall.net
}

\begin{abstract}
Characterizing of the mechanical behavior of sand under impact loading is necessary to estimate the power of explosive mines. Using Hopkinson pressure bars for its characterization only low and limited pressures can be applied. Therefore the experiments were performed on a high energy drop weight machine, which produces a high pressure up to several GPa much closer to real explosive processes. The triaxial stress state was realized within a thick steel ring. Measurements of forces and displacement of the punch and also the tangential strain of the ring were performed and used for the analysis of stresses. The influence of water contents and compaction of sand were investigated. Constitutive equations were derived for the different conditions.
\end{abstract}

\section{Introduction}

The behavior of soil under high rate loading is of fundamental importance related to a problem of a minesoil-structure interaction. There is a need for understanding the interaction of mine blast products with structures und the resulting loading and damage mechanisms inflicted by explosive blast and impact. Also the protection of the occupants of armored vehicles against the threat of antitank mines is the main goal of the protection technology. The anti-tank mines are generally buried in different soils. In addition to the depth of the mine, the soil type and moisture content have a significant influence on the mine action [1]. The knowledge of the effect of these parameters is of great importance for the estimation of the forces acting on the protected objects.

The aim of this investigation is the determination the effect of the different parameters on the mechanical behavior of sand under high impact loading rate. Many investigations were carried out on the split Hopkinson pressure bars [2-4]. The maximum pressure reached is about $350-400 \mathrm{MPa}$, is not representative for the pressure resulted from mine explosion. In the drop weight tests a very high pressure can be reached due to the drop of the heavy $600 \mathrm{~kg}$ weight, which produces a high impact energy of $3 \mathrm{~kJ}$ leading to pressures of more than $2000 \mathrm{MPa}$.

\section{Experimental}

For this investigation homogeneous model sand is selected. The grain size varies between 0.02 to $0.5 \mathrm{~mm}$. Figure 1 shows the distribution of grain size.

To determine the mechanical properties of the soil materials, dynamic compaction tests under impact loading are performed. The schematic construction of the compact test is shown in figure 2. The test material is put into a thick ring from high strength steel with a height of $8 \mathrm{~mm}$. The sample is loaded via two hard steel punches.

The experiments are performed on a drop weight machine with a falling weight of $600 \mathrm{~kg}$, figure 3 . The drop weight machine allows the simulation of the impact loading. The $600 \mathrm{~kg}$ weight falls from a preset height and impacts the upper punch, which compresses the soil sample. It results a high strain rate of $200 \mathrm{~s}^{-1}$.

During the experiment, the expansion of the steel ring is measured by strain gages fixed on the outer surface. The axial forces are measured through calibrated strain gages glued on punch. The measurement of the punch displacement is performed using an incremental optical encoder. These data are recorded with a high band width storage oscilloscope and are available for the evaluation.

\subsection{Evaluation of the compaction test}

In the compaction test, the axial stress $\sigma_{a}$ and the axial strain $\varepsilon_{a}$ are calculated as follows:

$$
\begin{gathered}
\sigma_{a}=\frac{F_{a}}{\pi \cdot R_{i}^{2}} \\
\varepsilon_{a}=\frac{\Delta h}{h}
\end{gathered}
$$

where $h$ is the height of the steel ring and $R_{i}$ the inner diameter, figure 4 .

The sample height is selected relatively small to the inner diameter. Therefore it may be assumed that the stress state and the deformation are homogeneous throughout the volume. The friction between sand and the inner cylindrical surface of steel ring is neglected for simplicity. The measured stresses are considered as the principal stresses.

$$
\begin{array}{cc}
\sigma_{1}=\sigma_{a} ; & \sigma_{2}=\sigma_{3}=\sigma_{r} \\
\varepsilon_{1}=\varepsilon_{a} ; & \varepsilon_{2}=\varepsilon_{3}=0
\end{array}
$$

Using the elastic theory of thick rings, the radial compression stress in the sand on the inner surface of the ring $\sigma_{r}$ can be calculated.

$$
\sigma_{r}=E \varepsilon_{\theta} \frac{R_{0}^{2}-R_{i}^{2}}{2 R_{i}^{2}}
$$

where $E$ is the Young's modulus of the ring material, $\varepsilon_{\theta}$ the measured tangential strain, $R_{0}$ the outer diameter and $R_{i}$ the inner diameter. 


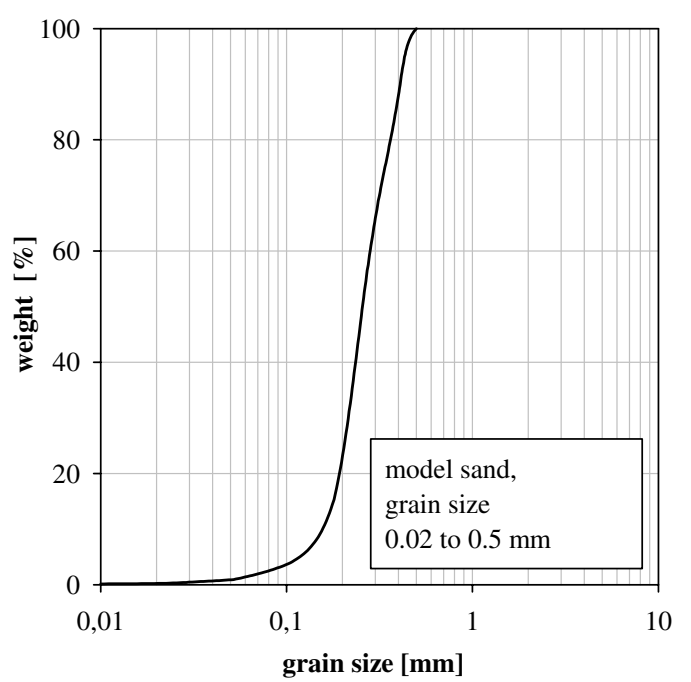

Fig. 1. Distribution of grain size.

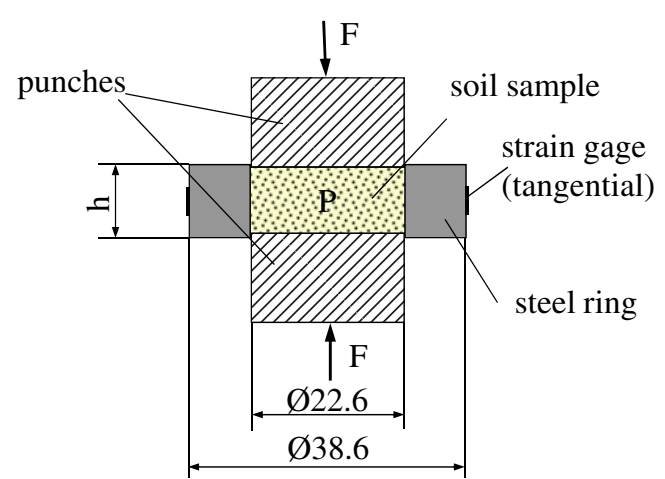

Fig. 2. Configuration of compaction test.

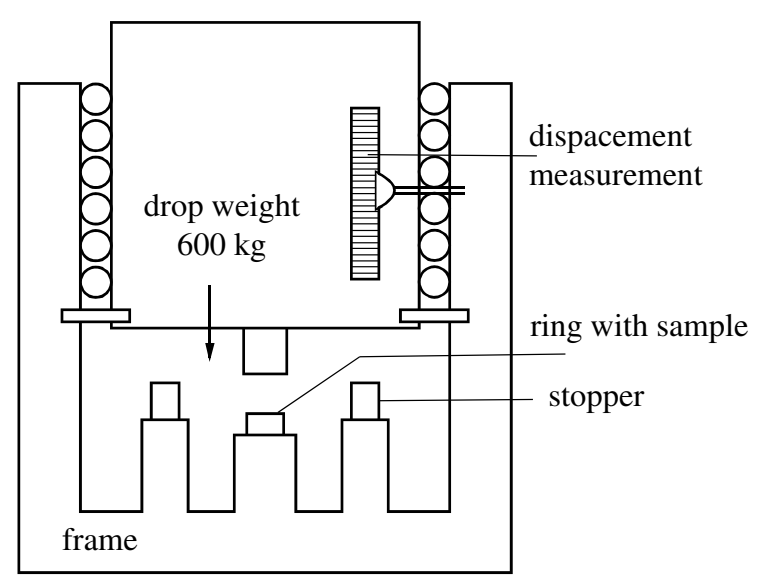

Fig. 3. Drop weight machine (schematic).

The hydrostatic pressure $P$ is the mean value of the principal stress:

$$
P=\frac{\sigma_{a}+2 \sigma_{r}}{3}
$$

The radial strain $\varepsilon_{r}$ is very small in comparison to the axial strain $\varepsilon_{a}$. Therefore the axial strain $\varepsilon_{a}$ can be considered equivalent to the compaction ratio $\theta$.

$$
\theta=\varepsilon_{a}
$$

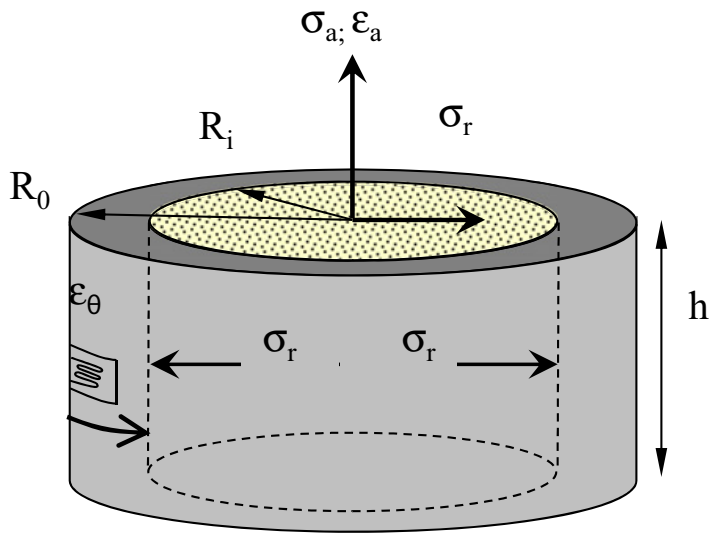

Fig. 4. Definitions of the compaction test parameters.

The maximum shear stress $\tau$ is calculated as

$$
\tau=\frac{\sigma_{a}-\sigma_{r}}{2}
$$

The ratio of radial stress $\sigma_{r}$ to axial stress $\sigma_{a}$ is defined as a factor of lateral thrust $\xi$.

$$
\xi=\frac{\sigma_{r}}{\sigma_{a}}
$$

\section{Experimental results}

The theoretical density of the sand is $2.66 \mathrm{~g} / \mathrm{cm}^{3}$. Due to the grain shape there are cavities between the grains. Therefore the initial density of a soil sample is always smaller than the theoretical density. For the tested sand a density of $1.36 \mathrm{~g} / \mathrm{cm}^{3}$ is measured in the normal dry case. Using shaking and hand compaction a second density of $1.56 \mathrm{~g} / \mathrm{cm}^{3}$ is adjusted. The investigations were performed at these two initial densities with different water contents; $0 \%, 5 \%, 10 \%$ and the saturated case nearly $20 \%$.

In figure 5 the results of the dynamically compacted sand are shown. The impact loading was applied on a drop weight machine with a mass of $600 \mathrm{~kg}$ and a falling height of $250 \mathrm{~mm}$. The resulting impact energy of $1500 \mathrm{~J}$ is focused on a sand volume of $2.84 \mathrm{~cm}^{3}$. The axial stress $\sigma_{a}$ was measured with an instrumented punch. The radial stress $\sigma_{r}$ was calculated from the measured tangential strain on the outer surface of the test ring according to equation (3). The hydrostatic pressure $\mathrm{P}$ was calculated from the stresses $\sigma_{a}$ and $\sigma_{r}$ according to equation (4).

The stress/strain and pressure/compaction curves can be described with exponential equations as shown in figure 6. For the impact energy of $1500 \mathrm{~J}$ the maximum axial stress shows the same values at all water contents, figure 7 . The radial maximum stress and the pressure exhibit low values for the dry sand and are increasing with increasing the water content. The maximum pressure is also depending on the initial density. In the lower density sand the maximum pressure is less than in the higher density sand, figure 8 . 


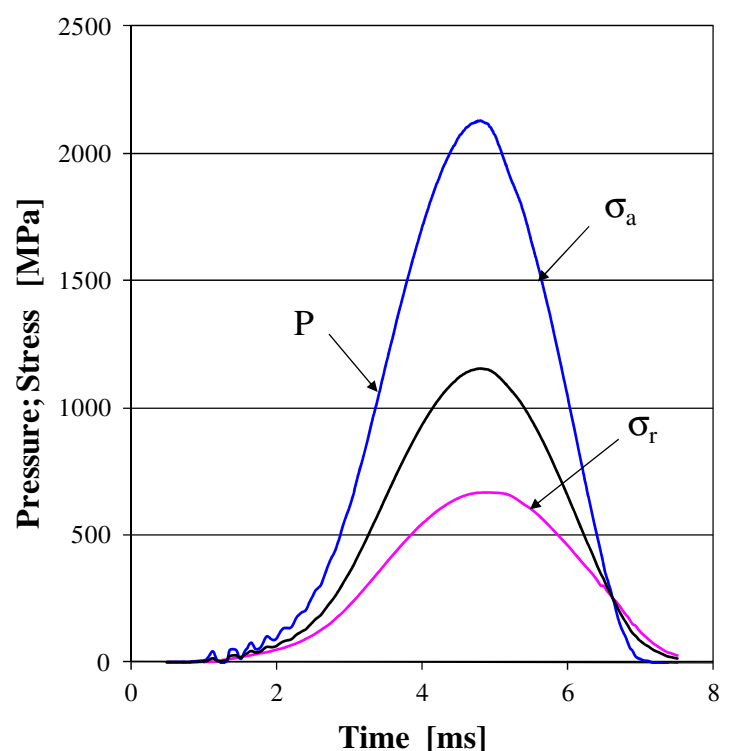

Fig. 5. Pressure and stress impulses in the tested dry sand with a initial density of $1.56 \mathrm{~g} / \mathrm{cm}^{3}$.

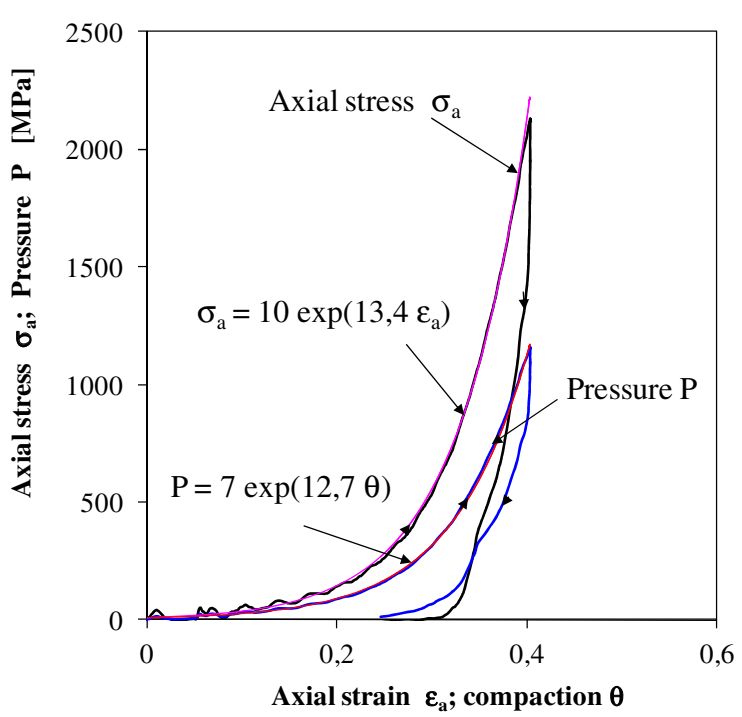

Fig. 6. Axial stress / axial strain and pressure / compaction curves of dry sand with initial density of $1.56 \mathrm{~g} / \mathrm{cm}^{3}$.

\section{Constitutive relations}

For the simulation of mine explosions in the sand, a constitutive equation for the sand is required, which not only combines two variables empirically, but also can describe the resulting hydrostatic pressure as a function of external loads, such as stresses, internal cohesions and friction. In this context, often the (static) Coulomb model is chosen, which is confirmed in its physical content of the dynamic results obtained here.

According to Bragov [5], the shear resistance of sand $\tau$ is dependent on the hydrostatic pressure $P$, on the cohesion between the sand particles $\mathrm{C}$ and on the tangent of the friction angle $\varphi$ :

$$
\tau \quad=C+P \cdot \tan \varphi
$$

The constant $\mathrm{C}$, the cohesion, is very small for sand.

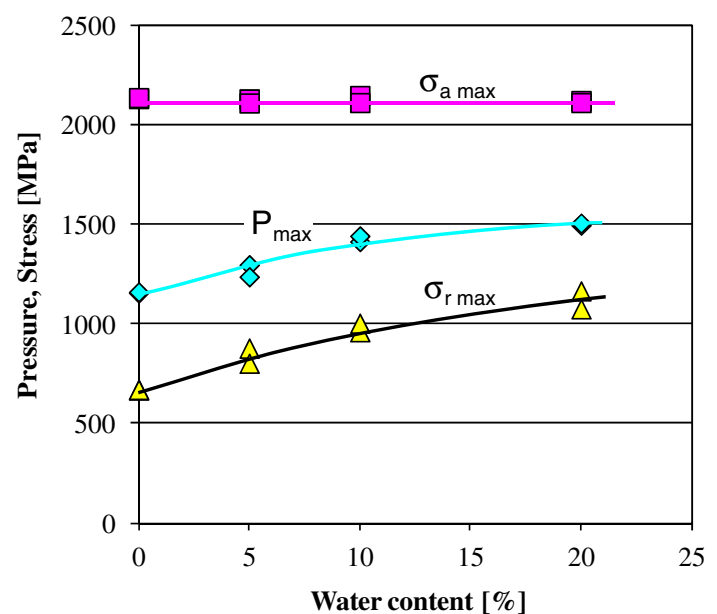

Fig. 7. Dependence of pressure and stresses maxima on moisture content.

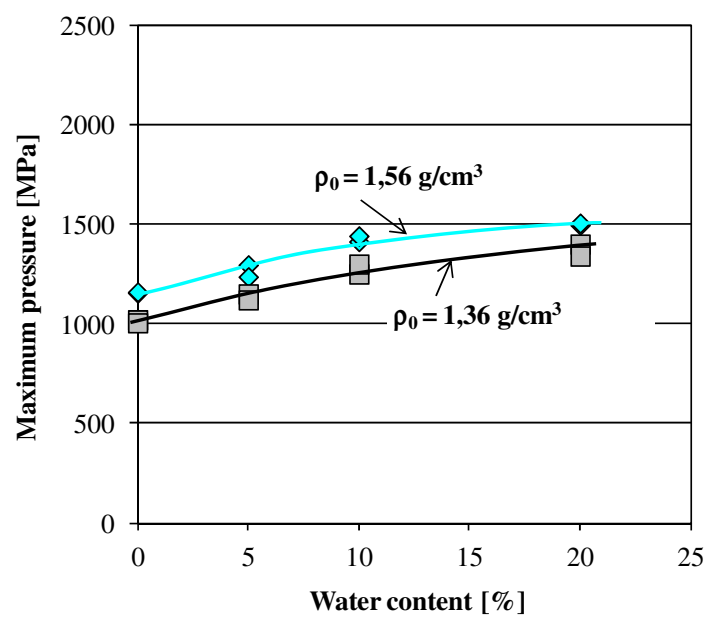

Fig. 8. Comparison of the reached maximum pressure at two different initial densities.

If the shear $\tau$ is replaced with equation (6) and the radial stress $\sigma_{r}$ is eliminated through equation (4), the hydrostatic pressure $P$ can be described in dependence of the axial stress $\sigma_{a}$ with the following equation:

$$
P=\frac{3 \sigma_{a}-4 C}{3+4 \tan \varphi}
$$

The constants $\mathrm{C}$ and $\varphi$ are dependent on the type of sand, grain form and size, and on water or clay content. The specific cohesion $\mathrm{C}$ is small and can be neglected. The internal friction angle $\varphi$ represents a resistance to the spread of the hydrostatic pressure. At low values of the internal friction angle $\varphi$, higher pressures are achieved. The factor of lateral thrust $\xi$, equation (7) is a scale for the transmission of external vertical loads into the lateral directions. At higher values of the factor of lateral thrust, the action of the mine explosion is stronger in the lateral directions of soil. Obviously equation (9) describes the real behavior of sand very well. An example for the comparison of the measured data with the fitted equation is shown in figure 9. In all tested cases, with the adjustment of the constants $\mathrm{C}$ and $\tan \varphi$, the physical content of equation (9) is expressed accurately. 


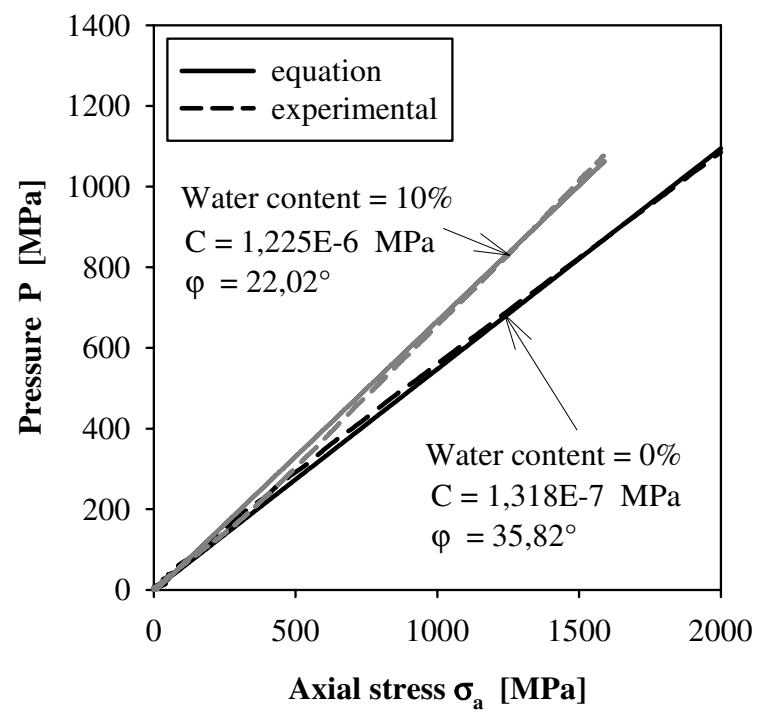

Fig. 9. Comparison of the measured pressure as a function of axial stress with the calculated data from equation (9) $\left(\rho_{0}=\right.$ $\left.1,56 \mathrm{~g} / \mathrm{cm}^{3}\right)$

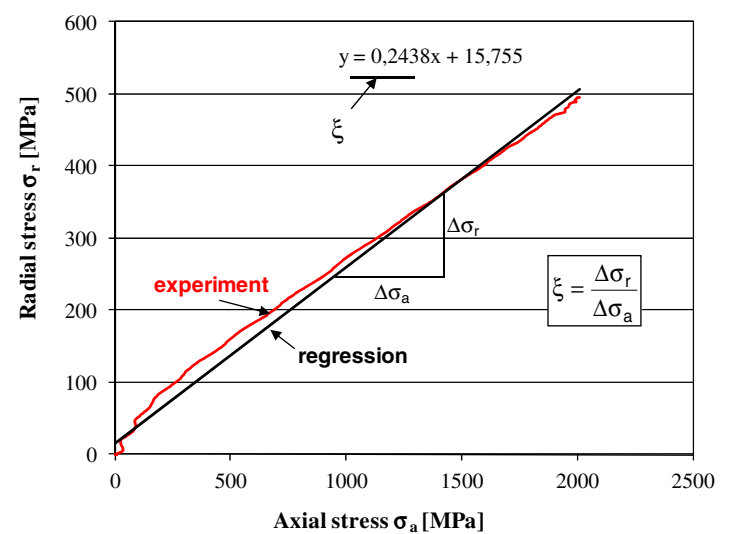

Fig. 10. Determination of the factor of lateral thrust (dry sand; $\left.\rho_{0}=1,56 \mathrm{~g} / \mathrm{cm}^{3}\right)$.

\section{Discussions}

An important result of the figure 9 is that, although the constants $\mathrm{C}$ and $\tan \varphi$ are dependent on water content, they show no changes during the dynamic compaction of sand. This simplifies the description of the constitutive behavior. The same constancy during the loading can be seen for the factor of lateral thrust $\xi$, figure 10. It varies also with the water content.

Under dynamic loading, the influence of the water content can be divided in two parts, figure 11. First part is the low water content part $(0 \%$ and $5 \%)$ which has high values of the friction factor $\tan \varphi$ and low values of factor of lateral thrust $\xi$. Second part is the high water part (10\% and more). It has low values of the friction factor $\tan \varphi$ and high values of factor of lateral thrust $\xi$. Between water content of $5 \%$ and $10 \%$, there is a sudden drop of the friction factor $\tan \varphi$ und a sudden rise of the lateral thrust $\xi$.

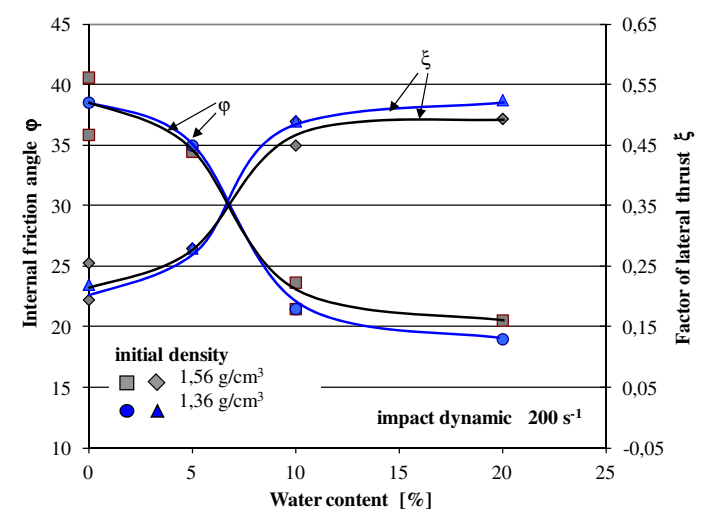

Fig. 11. Influence of water content on the constitutive parameters.

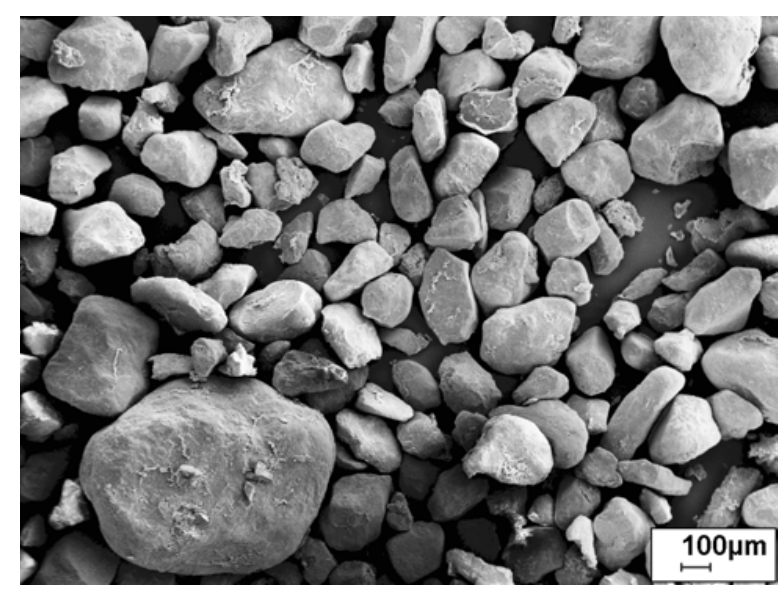

Fig. 12. Scanning electron microscopy picture of the sand before the test.

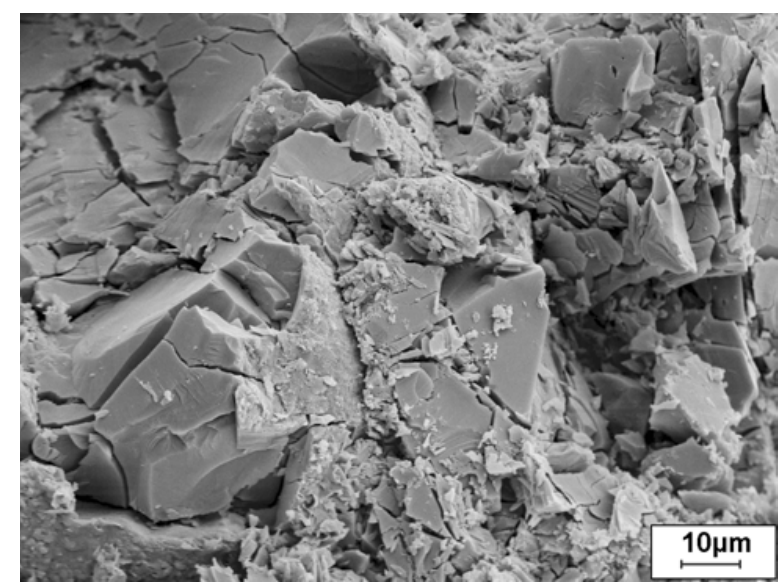

Fig. 13. Scanning electron microscopy picture of the sand after the test.

These results are different from the results of quasistatic loading. Under quasi-static loading the Parameters $\tan \varphi$ and $\xi$ are not influenced by the water content [6].

In figures 12 and 13 there are two photographs of the sand before and after the applied of the impact loading. Here it can be shown that the sand grains were destroyed in a brittle manner. The particles have initially 
a size of 1 to $10 \mu \mathrm{m}$. This may mean that the sand under high pressure is converted into powder. The density enhances towards the theoretical value. More compaction is not possible. Under repeated impacts no additional high hydrostatic pressures can be generated.

\section{Conclusions}

The compaction properties of a model sand were investigated under impact loading with a high energy drop weight machine up to a high axial compression stress of $2.2 \mathrm{GPa}$. It was proved that the water content influences the compaction properties, specially under impact loading.

\section{References}

1. L.W. Meyer, V. Mikulitsch, Proc. 11. Int. Symp. Interaction of the effects of Munitions with Structures, Mannheim, Germany. (2003)

2. B.E. Martin, W. Chen, Proc. ESM annual conf., Albuquerque, New Mexico, USA (2009)

3. H. Lu, H. Luo, R. Komaduri, Proc. ESM annual conf., Albuquerque, New Mexico, USA (2009)

4. M.E. Kabir, W.W. Chen, Proc. ESM annual conf., Indianapolis, Indiana, USA (2010)

5. A.M. Bragov, A.K. Lomunov, I.V. Sergeichev, K. Tsembelis, W.G. Proud, int. J. Impact Eng. 35 (2008) 967-976

6. S. Abdel-Malek, L.W. Meyer, internal Report of Nordmetall, not published (2006) 